\title{
New perspectives in cadmium toxicity: an introduction
}

\author{
Jean-Marc Moulis • Frank Thévenod
}

Received: 7 July 2010/Accepted: 7 July 2010/Published online: 15 July 2010

(C) Springer Science+Business Media, LLC. 2010

Why another series of reviews on cadmium (Cd) toxicity as so many reviews on the topic get regularly published in the literature? It is often stated that $\mathrm{Cd}$ is "natural," i.e., naturally present everywhere in air, water, soils, and foodstuffs. Yet, because of the now established fact that $\mathrm{Cd}$ is toxic, $\mathrm{Cd}$ emissions in the air have been constantly decreasing since the 1960s due to improved technology for the production, use, and disposal of $\mathrm{Cd}$ and $\mathrm{Cd}$-containing products. This may suggest that the health threat by $\mathrm{Cd}$ as a toxicant has receded. So, why bother about $\mathrm{Cd}$ and $\mathrm{Cd}$ associated health hazards?

But the industrial Cd consumption in the world has increased steadily from 18400 tons in 2003 to 20400 tons in 2007 (World Bureau of Metal Statistics,

J.-M. Moulis $(\square)$

Laboratoire de Chimie et Biologie des Métaux,

CEA-Grenoble, DSV, IRTSV, 17 rue des Martyrs,

38054 Grenoble Cedex 9, France

e-mail: jean-marc.moulis@cea.fr

J.-M. Moulis

CNRS UMR5249, Grenoble, France

J.-M. Moulis

Université Joseph Fourier, Grenoble, France

F. Thévenod $(\varangle)$

ZBAF, Department of Physiology and Pathophysiology,

University of Witten/Herdecke, Stockumer Str. 12,

58448 Witten, Germany

e-mail: frank.thevenod@uni-wh.de
Ware, United Kingdom; http://www.world-bureau.com/). As a chemical element, $\mathrm{Cd}$ cannot be degraded, and its concentration in the environment increases steadily, largely as a result of human activities. Recovery and safe removal of $\mathrm{Cd}$ from habitats is unrealistic in view of its ubiquitous occurrence in various forms in the environment. A major source of $\mathrm{Cd}$ in soils comes from the use of phosphate fertilizers for agricultural purposes. $\mathrm{Cd}$ accumulates along the food chain, in plants (root vegetables and shoots, such as rice, wheat, peanuts or cocoa, tobacco) and animals (offal, crustaceans and mollusks). Hence, $\mathrm{Cd}$ is an environmental contaminant of increasing importance. Over the last 15 years The Comprehensive Environmental Response, Compensation, and Liability Act (CERCLA) has permanently listed Cd as No 7 (out of 275) in its priority list of hazardous materials (ATSDR 2007). In 1993 the International Agency for Research on Cancer (IARC), which is part of the World Health Organization, classified $\mathrm{Cd}$ and $\mathrm{Cd}$-containing compounds as group-1 human carcinogens based on data obtained from human occupational exposure. Even though the carcinogenicity and toxicity of $\mathrm{Cd}$ at high doses have long been recognized, accumulating epidemiological evidence also suggests significant toxicity following long-term exposure to low doses of $\mathrm{Cd}$ which is not limited to cancer and affects more people than previously thought (see in particular Jarup and Akesson 2009; Satarug et al. 2010 for review). Even though maximal exposure values to limit chronic $\mathrm{Cd}$ toxicity may be reduced by legislation (CONTAM 
2009), the unavoidable increase of the Cd body burden during life can only be reasonably approached by proper knowledge of the molecular and cellular mechanisms at work in order to decrease uptake, accumulation, and to detoxify incorporated $\mathrm{Cd}$. Indeed, the current understanding of the biological effects of $\mathrm{Cd}$ and the existing knowledge of $\mathrm{Cd}$ induced diseases are mainly based on results obtained by exposure to high doses of the toxic metal with most obvious consequences. These data represent only "the tip of the iceberg" of Cd toxicity.

In this special issue of BioMetals on "New perspectives on Cd toxicity," experts deeply involved in Cd research as biologists, biochemists, physiologists, physicians and epidemiologists, have revisited and extended various facets of Cd toxicity. They focused on data which have been obtained with cutting-edge, wide ranging methods and they analyzed them with contemporary biological concepts. As a result, recurring actual topics of Cd toxicity and new fields of investigation emerge which forecast previously unsuspected health hazards of $\mathrm{Cd}$. We hope that the readers will share our enthusiasm to increase knowledge on the adverse effects of $\mathrm{Cd}$, not only to better combat the noxious effects of this metal on life, but to increase our understanding of basic principles in biology and toxicology.

In the first section, epidemiological and clinical aspects of Cd toxicity are reviewed by Nawrot et al. (2010) and Johri et al. (2010). Prozialeck and Edwards (2010) discuss novel early biomarkers of Cd nephrotoxicity, whereas Messner and Bernhard (2010) draw our attention to a neglected target of $\mathrm{Cd}$ toxicity, namely the cardiovascular system.

Chronic low dose exposure to $\mathrm{Cd}$ appears to be associated with an increasing spectrum of health hazards which is emphasized by Nawrot et al. (2010). According to current dogma, $\mathrm{Cd}$ damages the liver and the kidneys directly and bone indirectly via the kidneys. But recent evidence suggests that $\mathrm{Cd}$ also causes osteoporosis by directly targeting bone tissues. Diabetic populations seem to be more sensitive to renal toxicity induced by $\mathrm{Cd}$. Moreover, the potential causal links between $\mathrm{Cd}$ exposure and cardiovascular diseases (CVDs) or cancer (mainly lung, breast, prostate) are highlighted. Strikingly, environmental exposure to $\mathrm{Cd}$ increases mortality in a continuous fashion without measurable threshold effect, implying that there is no safe health-based limit value of exposure to the metal. In this context, the European Food and Safety Authority (CONTAM 2009) has established a tolerable weekly intake which is close to the average European $\mathrm{Cd}$ intake values and below those of many populations worldwide. Preventive measures aim to decrease the transfer of $\mathrm{Cd}$ from the environment and food chain to the body. They involve reduction of the availability of soil $\mathrm{Cd}$ to plants by neutralizing soil acidity. Further, because $\mathrm{Cd}$ is mainly transported by the iron uptake system of enterocytes, proper body iron status and iron availability in the diet reduce intestinal $\mathrm{Cd}$ absorption. Any preventive measures, however, call for further follow-up to demonstrate their efficacy.

Johri et al. (2010) describe the clinical characteristics of $\mathrm{Cd}$ toxicity in human populations, with emphasis on kidney disease. Whereas the effects of acute $\mathrm{Cd}$ toxicity depend on the type of exposure, by inhalation or ingestion, the major target organs of chronic toxicity are the skeleton, the lungs and, most importantly, the kidney. The latter accumulates Cdmetallothionein (MT) filtered from the circulation in the renal proximal tubule. The ensuing proximal tubular damage (and also some glomerular damage) results in proteinuria (mainly low molecular protein with microalbuminuria) as an early sign of a renal Fanconi syndrome. This contributes to the onset of bone disease, although $\mathrm{Cd}$ also directly targets the skeleton. Urinary low-molecular weight proteins can be used as diagnostic markers of "early" kidney damage but reversibility of kidney damage is already questionable at this stage. Progression of Cd-induced kidney damage may lead to chronic kidney disease and ultimately to renal failure. The occurrence of renal failure is promoted by diabetes, hypertension and co-exposure with other metals.

In their overview on early biomarkers of $\mathrm{Cd}$ toxicity, Prozialeck and Edwards (2010) focus on the kidney as the "sentinel of $\mathrm{Cd}$ exposure." They summarize the current state of Cd biomarker research in human populations, including indicators of kidney dysfunction which inform about the $\mathrm{Cd}$ body burden. Measurements of blood and urine $\mathrm{Cd}$ concentrations are of limited use to assess total body burden and damage to organs because $\mathrm{Cd}$ is sequestered before clear signs of intoxication appear. Some biomarkers reflect loss of tubular function and integrity, but by the time they appear in the urine, tissue injury is usually irreversible. Attractive novel developments in 
the area of kidney-derived early biomarkers include kidney injury molecule-1 (Kim-1) that appears early after toxic or ischemic tubular injury. Low levels of $\mathrm{Cd}$ affect serum concentrations and metabolism of glucose prior to the onset of kidney injury. Hence abnormal values of serum glucose and insulin may be early signs of $\mathrm{Cd}$ exposure.

Messner and Bernhard (2010) discuss the pathophysiological mechanisms of atherosclerosis. Various risk factors are responsible for CVDs, including age, male gender, diabetes, hypertension and smoking, which seem to coincide during the initiation of the disease. Cd now appears as a novel and independent risk factor contributing to CVDs. Interestingly, many of the risks factors for atherosclerosis are also associated with the "metabolic syndrome," suggesting links between $\mathrm{Cd}$ and the metabolic syndrome that remain to be explored. Cd stands out as a dominant noxious metal in tobacco smoke and it accumulates in endothelia and the arterial wall by several uptake routes. In endothelia, $\mathrm{Cd}$ activates various signaling processes which also contribute to induction of protective mechanisms, including increased synthesis of MT. The mechanism of atherosclerotic plaque formation is a complex and multi-stage process in which $\mathrm{Cd}$ can step in at different levels with proliferation of vascular smooth muscle, neo-angiogenesis, cell death, thrombosis, inflammation and decreased production of vasodilatory factors, such as NO. High Zn levels may protect against Cd-associated CVDs, as evidenced by preliminary epidemiological studies which await confirmation.

$\mathrm{Cd}$ is not needed by mammalian and most other living cells. Yet, the interference of $\mathrm{Cd}$ with a number of transport processes and with molecules requiring essential metals is amazing. The impact of $\mathrm{Cd}$ on membrane transport of metal cations and electrolytes and on intracellular processes are the topics of the second part of this special issue which is covered by Van Kerkhove et al. (2010), Thévenod (2010), and Moulis (2010).

Van Kerkhove et al. (2010) examine how $\mathrm{Cd}$ interferes with the ion transport homeostasis under conditions that are relevant to toxicity for the organism. The impact of $\mathrm{Cd}$ on the epithelial and renal handling of common inorganic ions, $\mathrm{H}_{2} \mathrm{O}$, and small organic molecules such as glucose and amino acids, is discussed in detail with regard to in vivo and in vitro effects. Moreover, acute and chronic intoxication by low (resulting in concentrations of 1-10 nM Cd in body fluids) or high doses of $\mathrm{Cd}$ (with concentrations of $1-5 \mu \mathrm{M} \mathrm{Cd}$ in body fluids) need to be considered to understand the pathophysiology of $\mathrm{Cd}$ toxicity in human populations. In particular, direct effects of $\mathrm{Cd}$ on transporter function (mostly inhibition or block due to exposure to high Cd concentrations) have to be distinguished from indirect effects. These may involve ROS formation (either as signaling or stress inducer molecules depending on the $\mathrm{Cd}$ concentration), activation of classical signal transduction pathways, membrane remodeling or protein maturation. All these processes may change transport activities which are difficult to interpret if not complemented by molecular studies with $\mathrm{Cd}$ concentrations and exposure times that are as close as possible to in vivo situations.

Most mechanisms of $\mathrm{Cd}$ toxicity are activated once the metal has entered the cells. Some proteins mediating membrane transport of essential metals (such as iron, zinc and manganese) and Cd have been recently identified, in particular the divalent metal transporter 1 (DMT1) or the Zrt- and Irt-related proteins 8 and 14 (ZIP8/14). But they do not account for all $\mathrm{Cd}$ movements in, out, and within cells. A survey of the less clearly evidenced transport sytems that may contribute to $\mathrm{Cd}$ traffic is provided by Thévenod (2010). The title of the review (Catch me if you can!) emphasizes the elusive nature of $\mathrm{Cd}$ ions which may compete with biologically essential metals for transport, but may also block ion fluxes (for instance through $\mathrm{Ca}^{2+}$ channels), or use alternative uptake systems as $\mathrm{Cd}$ complexes (e.g., uptake of Cd-MT via megalin receptor-mediated endocytosis) to distribute throughout the body. By correlating transporter expression and Cd-resistance or -toxicity, multiple studies have inferred that $\mathrm{Cd}$ is transported by a whole collection of transporters. Direct proof of $\mathrm{Cd}$ transport, however, needs to be obtained by applying electrophysiological, radiotracer, or Cdsensitive fluorescent dye techniques in an heterologous expression system prior to in vivo verification.

The molecular and cellular mechanisms of $\mathrm{Cd}$ toxicity are intimately linked to the cellular control of most biological cations, including transition metals. Why are the effects of $\mathrm{Cd}$ on cellular and tissue functions so complex and manifold? This question is addressed by Moulis (2010) who highlights the fact 
that many biomolecules need cations to fulfill their function or bind them non-specifically. $\mathrm{Cd}$ can interfere with the physiological metals by replacing them, but, although many metal-binding proteins have been shown to bind $\mathrm{Cd}$ in vitro, such evidence is usually lacking in the physiological environment of the cell. Mere replacement of one (physiological) cation by another ( $\mathrm{Cd}$, the toxic one) cation at specific sites falls short of explaining all available observations regarding $\mathrm{Cd}$ toxicity. Although direct effects of $\mathrm{Cd}$ on metalloproteins can partly explain $\mathrm{Cd}$ toxicity, the impact of $\mathrm{Cd}$ on signaling processes (ROS, $\mathrm{Ca}^{2+}$, etc.) strongly affects the homeostasis of essential metals as well. Examples of mechanistic interactions between cellular metal handling and $\mathrm{Cd}$ toxicity abound, and the examination of the detailed homeostatic pathways of biological essential metals suggests new $\mathrm{Cd}$ targets that remain to be analyzed.

Inside the cell, $\mathrm{Cd}$ elicits a number of reactions which may lead to death or stress adaptation, survival and cancer. These processes are mediated by signaling pathways which induce up-regulation of various stressinducing or protective molecules. In the third section, Sabolic et al. (2010) emphasize the pleiotropic role of the metal-binding protein MT in Cd toxicity and survival, Cuypers et al. (2010) and Kitamura and Hiramatsu (2010) describe redox and endoplasmic reticulum (ER) stress signaling evoked by $\mathrm{Cd}$, respectively, whereas Hartwig (2010) provides novel insights into the mechanisms of $\mathrm{Cd}$ carcinogenesis.

Probably the most pivotal biomolecule to consider when dealing with Cd toxicity is MT. Sabolic et al. (2010) provide a detailed review on current knowledge, controversies and open issues concerning MT. The different localization and physiological functions of MTs, including those of the metal-free (apo-MT) and metal-bound (holo-MT) forms, in various organs and intracellular organelles impact the redox and energy status of the cells. Knowledge of age, sex and strain differences in mammalian studies is particularly relevant to understand the phenomenology of $\mathrm{Cd}$ toxicity, but this has been neglected so far. The double-edged role of MT in organ toxicity is underlined by its involvement as a protein preventing (antioxidant, chelator) and mediating (carrier) $\mathrm{Cd}$ toxicity. To underscore open issues and future directions to pursue, the authors complement the extensive survey of the literature on the roles of MT in $\mathrm{Cd}$ toxicity by experimental data from their own laboratory.

Though it is uncontested that $\mathrm{Cd}$ induces formation of reactive oxygen/nitrogen species (ROS/RNS), $\mathrm{Cd}$ is not redox active. Cuypers et al. (2010) describe the mechanisms and sources of ROS formation in the presence of $\mathrm{Cd}$, which involve catalysis by uncontrolled redox active metals, enhancement of ROS formation by the mitochondrial respiratory chain and induction of NADPH oxidase (NOX) enzymes. The latter mechanism exemplifies the key role of ROS in cellular adaptation to $\mathrm{Cd}$. Persistent NOX expression results from an amplification loop triggered by ROS and ROS-sensitive transcription factors, whereas a defense loop is mediated by ROS which also trigger up-regulation of protective antioxidative mechanisms, thus promoting repair and survival, but also malignancy. The duality of ROS/RNS in the balance between positive and negative feed-back loops and as physiological signaling messengers versus toxic molecules is a major feature of the role of ROS/ RNS in Cd toxicity.

Kitamura and Hiramatsu (2010) review recent evidence for the involvement of ER stress signaling and the unfolded protein response (UPR) in $\mathrm{Cd}$ apoptosis in vivo and in vitro. Cd-induced cellular stress disturbs proper folding of membrane and secreted proteins in the ER and triggers UPR which determines whether damage control or death by apoptosis occurs. Whereas the ATF6 and IRE1 pathways of the UPR cooperatively promote $\mathrm{Cd}$ apoptosis by recruiting CHOP and JNK, the PERK pathway prevents it. ROS may operate up- or downstream of ER stress, but in the kidney proximal tubule and bronchial epithelial cells Cd induces ROS formation which elicits ER stress. Specifically, the superoxide radical anion, but not $\mathrm{H}_{2} \mathrm{O}_{2}$, appears to selectively trigger activation of the pro-apoptotic branches of the UPR induced by Cd. Apart from the ER stress-UPR signaling, other pathways with proand anti-apoptotic potential are also activated by $\mathrm{Cd}$ and ROS, namely calcium and NF-kB signaling. Interestingly, they are known to cross-talk with the ER stress-UPR axis. Hence, it remains to be determined how pro-apoptotic and adaptive responses to $\mathrm{Cd}$ are linked to the UPR, but the observation that ER stress participates in $\mathrm{Cd}$ toxicity suggests novel strategies for the prevention and therapy of $\mathrm{Cd}$ - 
induced toxicity using chemicals with chaperone function.

Cd causes cancer of the lungs, kidney and prostate, and possibly female breast and endometrial cancer. Hartwig (2010) reviews the underlying molecular mechanisms. Cd is not directly mutagenic but it is a strong co-mutagen. Persistent DNA damage is due to indirect processes, such as increased ROS formation (partly due to $\mathrm{Cd}$ inhibition of cellular anti-oxidative defense), interference with the DNA damage response system, and epigenetic changes in DNA methylation patterns leading to genomic instability. ROS signaling may also induce proliferation and/or redox-sensitive transcription factors that promote carcinogenicity. However, DNA damage is observed at much lower $\mathrm{Cd}$ concentrations than needed to induce oxidative DNA base modifications, indicating that low Cd impairs almost all major DNA repair systems. Why are these mechanisms particularly sensitive to $\mathrm{Cd}$ ions? They are controlled by metalbinding proteins, particularly by Zn-finger proteins (and to some extent by calcium-binding proteins) which are involved in DNA binding and proteinprotein interactions. Some of these protein domains are particularly sensitive to $\mathrm{Cd}$ which inhibits or damages DNA repair enzymes, cell cycle control proteins, pro-apoptotic and tumor-suppressing proteins (e.g., PARP-1, p53), Zn-containing transcription factors, enzymes mediating DNA methylation, and also E-cadherin. Why are specific organs affected by cancer? They accumulate $\mathrm{Cd}$ (e.g., via uptake pathways and MT upregulation), develop Cd resistance (e.g., by increased ROS tolerance) or rally cellular pathways opposing apoptosis. A combination of all these processes leads to hypermutability, reduces growth and cell cycle control and induces resistance to apoptosis thus contributing to cancer initiation and progression.

Now that the health hazards of Cd exposure are made out, the task lying ahead is to fully explain the cellular impact of this insidious toxic compound, particularly at low exposure doses. Although not an easy task, this is the challenge that scientists will have to tackle to come up with useful solutions to circumvent the deleterious effects of $\mathrm{Cd}$ on the environment and on human health.

We, as guest editors, would like to acknowledge the opportunity given by BioMetals to gather these insightful reviews by authors who contributed to many new facets of $\mathrm{Cd}$ toxicology. These thanks must be extended to referees who significantly improved the general quality of the submitted manuscripts. BioMetals has provided ample room for presenting these reviews in the most attractive way with unrestricted color illustrations and careful production. We hope this attractiveness will encourage readers of various backgrounds to get useful information in the most enjoyable manner, and to develop new ideas for studies on Cd toxicity.

Acknowledgments The laboratory of F.T. is supported by Deutsche Forschungsgemeinschaft (TH 345/10-1 and 11-1).

\section{References}

ATSDR (2007) Agency for toxic substance and disease registry, U.S. toxicological profile for cadmium. Department of Health and Humans Services, Public Health Service, Centers for Disease Control, Atlanta

CONTAM (2009) Scientific opinion of the panel on contaminants in the food chain on a request from the European Commission on cadmium in food. EFSA J 980:1-139

Cuypers A, Plusquin M, Remans T et al (2010) Cadmium stress: an oxidative challenge. Biometals. doi:10.1007/ s10534-010-9329-x

Hartwig A (2010) Mechanisms in cadmium-induced carcinogenicity: recent insights. Biometals. doi:10.1007/s10534010-9330-4

Jarup L, Akesson A (2009) Current status of cadmium as an environmental health problem. Toxicol Appl Pharmacol 238:201-208

Johri N, Jacquillet G, Unwin R (2010) Heavy metal poisoning: the effects of cadmium on the kidney. Biometals. doi:10.1007/s10534-010-9328-y

Kitamura M, Hiramatsu N (2010) The oxidative stress: endoplasmic reticulum stress axis in cadmium toxicity. Biometals. doi:10.1007/s10534-010-9296-2

Messner B, Bernhard D (2010) Cadmium and cardiovascular diseases: cell biology, pathophysiology, and epidemiological relevance. Biometals. doi:10.1007/s10534-0109314-4

Moulis JM (2010) Cellular mechanisms of cadmium toxicity related to the homeostasis of essential metals. Biometals. doi:10.1007/s10534-010-9336-y

Nawrot TS, Staessen JA, Roels HA et al (2010) Cadmium exposure in the population: from health risks to strategies of prevention. Biometals. doi:10.1007/s10534-010-9343-Z

Prozialeck WC, Edwards JR (2010) Early biomarkers of cadmium exposure and nephrotoxicity. Biometals. doi:10.1007/ s10534-010-9288-2

Sabolic I, Breljak D, Skarica M et al (2010) Role of metallothionein in cadmium traffic and toxicity in kidneys and other mammalian organs. Biometals. doi:10.1007/s10534010-9351-z 
Satarug S, Garrett SH, Sens MA et al (2010) Cadmium, environmental exposure, and health outcomes. Environ Health Perspect 118:182-190

Thévenod F (2010) Catch me if you can! Novel aspects of cadmium transport in mammalian cells. Biometals. doi:10.1007/s10534-010-9309-1
Van Kerkhove E, Pennemans V, Swennen Q (2010) Cadmium and transport of ions and substances across cell membranes and epithelia. Biometals. doi:10.1007/s10534-0109357-6 